ARTICLE

\title{
14-3-3 proteins regulate Tctp-Rheb interaction for organ growth in Drosophila
}

Thao Phuong Le ${ }^{1}$, Linh Thuong Vuong ${ }^{1, \dagger}$, Ah-Ram Kim', Ya-Chieh Hsu² \& Kwang-Wook Choi

14-3-3 family proteins regulate multiple signalling pathways. Understanding biological functions of 14-3-3 proteins has been limited by the functional redundancy of conserved isotypes. Here we provide evidence that 14-3-3 proteins regulate two interacting components of Tor signalling in Drosophila, translationally controlled tumour protein (Tctp) and Rheb GTPase. Single knockdown of $14-3-3 \varepsilon$ or $14-3-3 \zeta$ isoform does not show obvious defects in organ development but causes synergistic genetic interaction with Tctp and Rheb to impair tissue growth. 14-3-3 proteins physically interact with Tctp and Rheb. Knockdown of both 14-3-3 isoforms abolishes the binding between Tctp and Rheb, disrupting organ development. Depletion of 14-3-3s also reduces the level of phosphorylated S6 kinase, phosphorylated Thor/4E-BP and cyclin E (CycE). Growth defects from knockdown of 14-3-3 and Tctp are suppressed by CycE overexpression. This study suggests a novel mechanism of Tor regulation mediated by 14-3-3 interaction with Tctp and Rheb.

\footnotetext{
${ }^{1}$ Department of Biological Sciences, Korea Advanced Institute of Science and Technology, 291 Daehak-ro, Yuseong-gu, Daejeon 305-701, Korea.

${ }^{2}$ Department of Stem Cell and Regenerative Biology, Harvard University, Sherman Fairchild 358A, 7 Divinity Avenue Cambridge, Massachusetts 02138, USA. $\dagger$ Present address: Department of Developmental and Regenerative Biology and Graduate School of Biomedical Sciences, Icahn School of Medicine at Mount Sinai, New York, New York 10029, USA. Correspondence and requests for materials should be addressed to K.-W.C. (email: kchoi100@kaist.ac.kr).
} 
T he Tor signalling pathway is important for the regulation of cell growth, proliferation, survival and metabolism in eukaryotes. Abnormal regulation of Tor signalling can cause various diseases including cancer and diabetes. Tor is the catalytic kinase subunit of the TORC1 complex that consists of several regulatory components such as Raptor and Pras 40. The TORC1 complex integrates extracellular signalling and intracellular processes ${ }^{1}$. Growth factors like insulin activate Pi3k, leading to Akt activation. Akt is known to phosphorylate tuberous sclerosis complex 2 (Tsc2) to inhibit its GTPaseactivating protein (GAP) activity for Rheb GTPase ${ }^{2,3}$. Hence, inactivation of the Tsc1/2 complex promotes Rheb function to activate Tor kinase ${ }^{4}$. A key role of Tor kinase is to stimulate protein synthesis for cell growth by phosphorylating ribosomal S6 kinase (S6k) and Thor (Drosophila 4E-BP). Lack of S6k results in a reduction of the cell size but not the cell number ${ }^{5}$. In Drosophila, Rheb and Tor kinase mutants show cell cycle arrest ${ }^{6,7}$. Loss of Tor in imaginal discs leads to a reduced cell proliferation and cyclin $\mathrm{E}(\mathrm{CycE})$, the S-phase regulator. Further, the cell cycle arrest in Tor mutants can be rescued by overexpressing $\mathrm{CycE}^{7}$, indicating that Tor signalling is required for the regulation of the CycE level, thus affecting cell proliferation.

Recent studies have shown that translationally controlled tumour protein (Tctp) is involved in Tor signalling ${ }^{8-13}$. Tctp is a family of evolutionarily conserved proteins involved in a number of fundamental processes, including cell proliferation, apoptosis and DNA damage control ${ }^{14-17}$. Tctp is upregulated in cancer cells, and its reduction results in the reversion of tumour phenotypes, implicating its role in tumorigenesis and tumour reversion ${ }^{14,18,19}$. In Drosophila, Tctp is required for organ growth by promoting Rheb function for Tor signalling as a guanine nucleotide exchange factor ${ }^{8}$. The role of Tctp in Tor signalling has also been reported in other organisms ${ }^{9-13}$. The function of Tctp family proteins in growth regulation seems to be conserved since Drosophila growth defects by Tctp knockdown can be restored by human Tctp $^{8}$. Remarkably, functional conservation has also been found between animals and plants. For instance, Drosophila Tctp rescues embryonic lethality and cell proliferation defects in Arabidopsis Tctp (AtTctp) loss-of-function mutant. Consistent with this interspecies complementation, Drosophila Rheb not only interacts with Tctp but also with the plant AtTctp $^{13}$. Since Rheb is essential for Tor activation, it is important to understand how the interaction between Tctp and Rheb is regulated.

Interestingly, 14-3-3 proteins interact with multiple regulators of Tor signalling such as Tsc2, Pras40 and Raptor in mammalian cells ${ }^{20-24} \cdot 14-3-3$ proteins are conserved adaptor molecules that control diverse signalling pathways ${ }^{25,26}$, but their function in vivo has not been well characterized. Loss-of-function studies in Drosophila indicated that Pras 40 is only required in ovary but not in other tissues ${ }^{27}$. Thus, it is important to identify physiologically critical functions of $14-3-3 \mathrm{~s}$ and their interacting proteins in animal models. In Drosophila, 14-3-3 proteins are encoded by two genes, $14-3-3 \varepsilon$ and $14-3-3 \zeta$ (also named leo for leonardo) ${ }^{28}$. Null mutations of either $14-3-3 \varepsilon$ or $14-3-3 \zeta$ lead to embryonic lethality. However, some 14-3-3e homozygous mutants are viable, because lack of $14-3-3 \varepsilon$ is compensated by elevated level of $14-3-3 \zeta$ protein $^{29}$. Drosophila 14-3-3 genes participate in Ras/Mapk signalling and neuronal differentiation $^{30,31}$. They are also known to modulate FoxOmediated apoptosis ${ }^{32}$, Hippo signalling 33,34 , and cell cycle regulation in syncytial nuclear division during embryogenesis ${ }^{35}$, but their roles in Tor signalling have not been studied.

In this study, we identify 14-3-3 proteins as binding partners for Tctp and Rheb. 14-3-3 genes show strong genetic interaction with Tctp and Rheb. 14-3-3 proteins physically interact with Tctp and Rheb. We provide evidence that 14-3-3 proteins are required for promoting the interaction between Tctp and Rheb. Loss of both 14-3-3 isoforms critically impairs organ development, and such defects are strongly suppressed by CycE. Our data suggest that 14-3-3 proteins regulate the interaction between Tctp and Rheb for organ growth, providing novel insights into their functions in Tor signalling.

\section{Results}

Tctp and Rheb interact genetically with 14-3-3 genes. We have attempted to identify new genes interacting with Tctp by searching for genetic modifiers of the reduced eye phenotype caused by Tctp RNA interference (RNAi) using Gal4/UAS system $^{36}$. UAS-Tctp RNAi and eyeless (ey)-Gal4 were used to induce Tctp silencing mainly in undifferentiated cells of eye imaginal disc ${ }^{8}$. Knockdown of either $14-3-3 \varepsilon$ or $14-3-3 \zeta$ alone did not show gross abnormalities in the eye (Fig. 1b,c). In contrast, 14-3-3e RNAi strongly enhanced the effects of Tctp knockdown in the eye, resulting in greatly reduced eyes (Fig. 1g) compared with a mild reduction by Tctp RNAi alone (Fig. 1f). We tested

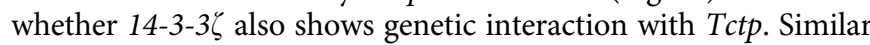

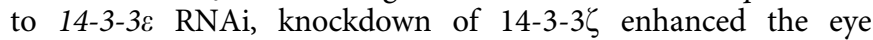

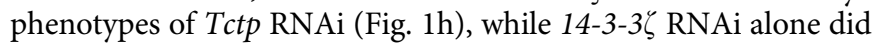
not show obvious defects in the eye (Fig. 1c). 14-3-3E or 14-3-36 mutant clones generated in eye and wing discs also appeared to be similar in the pattern of Elav (neuron-specific nuclear marker) and Discs-large (Dlg; cell membrane marker), respectively, compared with the surrounding wild-type tissues (Supplementary Fig. 1). To further confirm the synergistic genetic interaction between Tctp and 14-3-3 genes, we tested whether Tctp RNAi eye phenotypes can be enhanced by 14-3-3 mutations, $14-3-3 \varepsilon^{22 B 10}$ and $14-3-3 \zeta^{07103}$. Although $14-3-3 \varepsilon^{j 2 B 10} /+$ and $14-3-3 \zeta^{07103} /+$ heterozygotes did not show any obvious eye defect (Fig. 1d,e), these heterozygous conditions led to considerable enhancement of the Tctp RNAi eye phenotype (Fig. 1i,j).

We then examined whether the genetic interaction between Tctp and 14-3-3 genes can also be seen in another organ. When nubbin (nub)-Gal4 was used to drive Tctp RNAi in developing wing disc, it resulted in consistent reduction and mild wrinkling

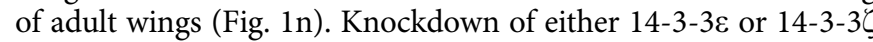
did not reduce the wing size (Fig. $11, \mathrm{~m}$ ), although it showed partial loss of crossveins (Fig. 11). However, 14-3-3 RNAi strongly enhanced the effects of Tctp knockdown, causing reduction and severe wrinkling of wings (Fig. 1o,p). We performed similar genetic tests with two additional independent Tctp RNAi lines and obtained similar results, as shown in Supplementary Fig. 2. These RNAi lines driven by ey-Gal4 or nub-Gal4 resulted in reduced eye and wing, respectively (Supplementary

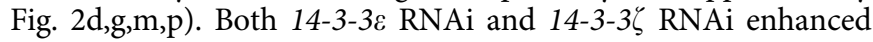
the eye/wing phenotypes caused by Tctp RNAi (Supplementary Fig. 2d-i,m-r). These data indicate that 14-3-3 and Tctp genetically interact in both eye and wing development.

In Tor signalling, Tsc1/2 complex functions as GAP towards Rheb $^{37,38}$. Thus, we tested whether 14-3-3 genes genetically interact with $T s c 2$ in the wing model. Control single knockdown of either isoform of 14-3-3 in the wing using nub-Gal4 did not affect the wing size (Fig. 2b,c), as also shown in Fig. 1. Knockdown of Tsc2 in the wing led to $19.5 \pm 0.05 \%$ tissue enlargements by overgrowth (Fig. 2d,j, $n=10$ ). Remarkably, silencing of either $14-3-3 \varepsilon$ or $14-3-3 \zeta$ strongly inhibited the wing growth caused by Tsc2 RNAi, even showing severe undergrowth of wings (Fig. 2e,f). Next, we examined whether 14-3-3 genes might also interact genetically with Rheb. Overexpression of Rheb by nub-Gal4 induced $23 \pm 0.06 \%$ increase in wing size (Fig. 2g,j, $n=10$ ). Knockdown of $14-3-3 \varepsilon$ inhibited the wing overgrowth 
caused by Rheb overexpression (Fig. 2h). Enlarged wing by Rheb

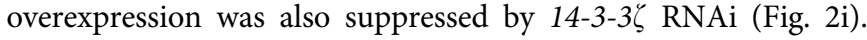
This suppression by either one of 14-3-3 RNAi resulted in mild curling at the wing margin, but the wing size was consistently reduced to that of the wild-type control (Fig. 2j). We also tested the effects of 14-3-3 RNAi on cell size. Because each wing cell generates a single hair, the hair density within a given area provides an approximate estimation of cell size. Knockdown of either $14-3-3 \varepsilon$ or $14-3-3 \zeta$ did not noticeably affect the wing hair density (Fig. $2 \mathrm{k}-\mathrm{m}$ ). The hair density was decreased by $13.1 \pm 0.58 \%$ in $n u b>T s c 2$ RNAi wings (Fig. $2 \mathrm{n}, \mathrm{t}, n=10$ ) and by $14.5 \pm 1.83 \%$ in $n u b>$ Rheb wings (Fig. 2q,t, $n=10$ ), suggesting an increase in cell size. The decreased wing hair density by Tsc2 silencing or Rheb overexpression was suppressed by knockdown of either $14-3-3 \varepsilon$ (Fig. $20, \mathrm{r}, n=10$ ) or $14-3-3 \zeta$ (Fig. 2p,s, $n=10$ ).

Tctp and Rheb physically interact with 14-3-3 proteins. Strong genetic interaction of 14-3-3s with Tctp and Rheb suggests that they may function together to regulate organ development. Hence, we checked the expression of Tctp and Rheb in wing and eye discs from wild-type third instar larvae. For cellular localization of Rheb, we generated an anti-Rheb antibody which specifically detected Rheb protein in tissues (Supplementary Fig. 3a-f). Consistent with the physical interaction between Tctp and $\mathrm{Rheb}^{8}$, both proteins showed an overlapping expression pattern in eye disc (Supplementary Fig. 3g-i). Interestingly, loss of Tctp in mutant clones resulted in a reduction of the Rheb level in both eye and wing discs (Fig. 3c,g). We used an anti-14-3-3 antibody that specifically recognizes $14-3-3$ s but does not distinguish $14-3-3 \varepsilon$ and $14-3-3 \zeta$ on western blot ${ }^{32}$. Immunostaining of wing discs with this antibody showed ubiquitous expression of 14-3-3s, but its level was reduced by 14-3-3e RNAi (Supplementary Fig. 3j-1), indicating the specificity of this antibody. Unlike Rheb, loss of Tctp did not cause obvious changes in the level of 14-3-3s (Fig. 3d,h).

Strong genetic interaction between Tctp and 14-3-3s, raised a possibility that they might physically interact. Co-immunoprecipitation (Co-IP) tests indicated that 14-3-3E and $14-3-3 \zeta$ are associated with Tctp in a protein complex in S2 cell extracts (Fig. 4a). GST pull-down assays using bacterially expressed fusion proteins indicate that both 14-3-3E and 14-3-36 bind to Tctp protein (Fig. $4 \mathrm{~b}$ ). These data suggest that 14-3-3e and $14-3-3 \zeta$ directly interact with Tctp in a protein complex. As shown in Fig. 1, 14-3-3 genes show genetic interaction with Rheb as well as Tctp. Because Tctp and Rheb are directly associated, it is plausible that Rheb might also form a complex with 14-3-3. Co-IP assays using S2 cell extracts showed that Rheb co-immunoprecipitates with $14-3-3 \varepsilon$ and $14-3-3 \zeta$ (Fig. 4c). Remarkably, Rheb was also pulled down by GST-14-3-3 $\varepsilon$ and

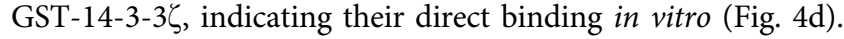
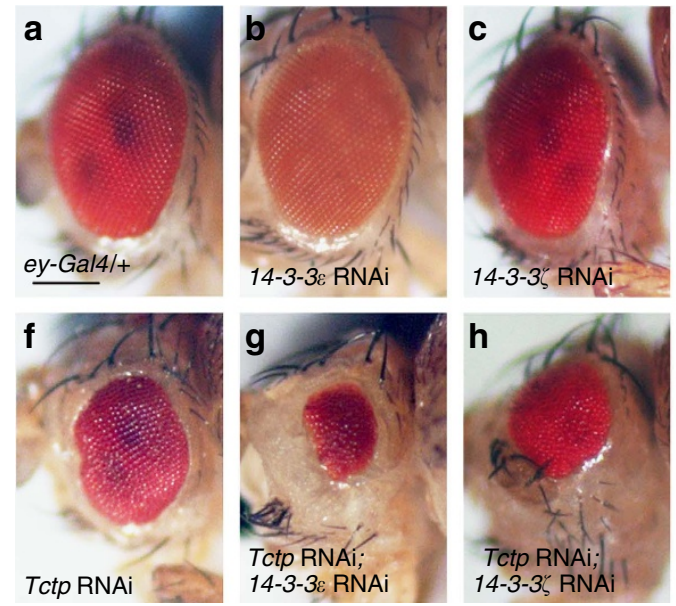
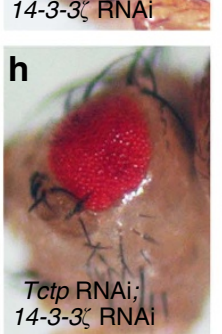
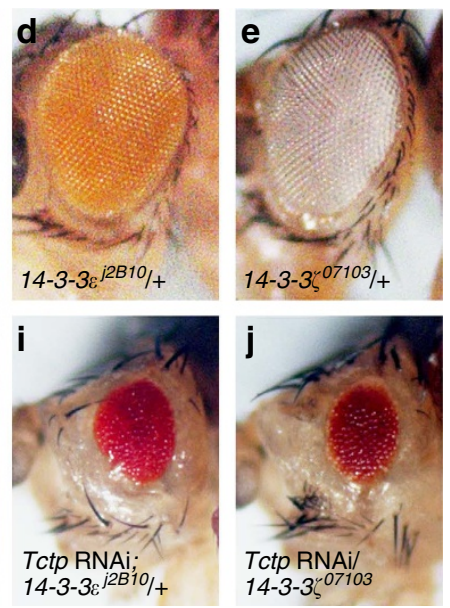
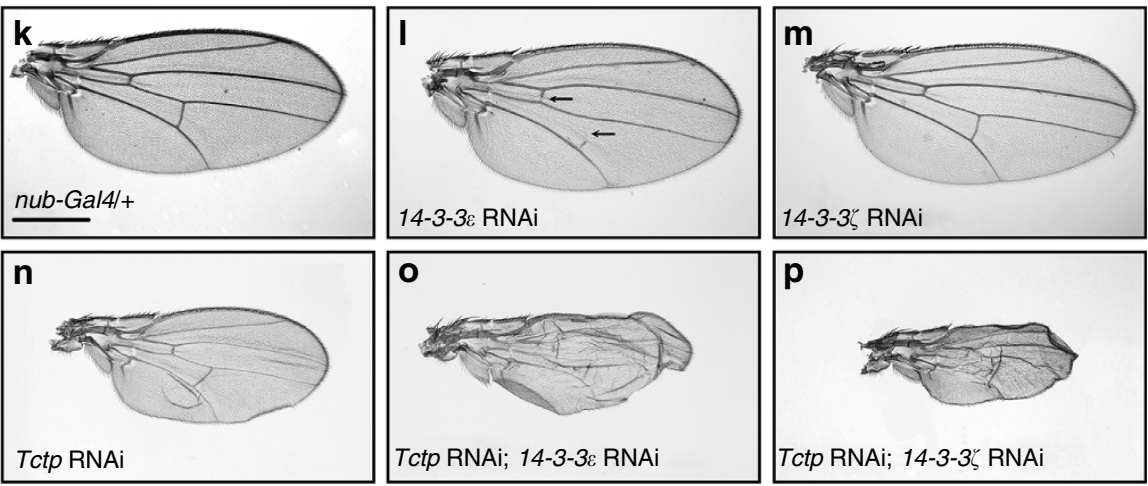

Figure 1 | Tctp and Rheb interact genetically with 14-3-3. (a-j) Genetic interaction between Tctp and 14-3-3 in eye. (a) Adult fly with one copy of

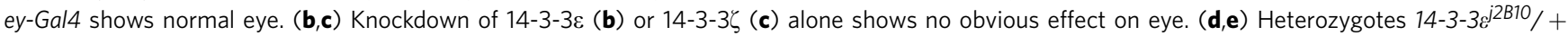
(d) or $14-3-3 \zeta^{07103} /+(\mathbf{e})$ show normal eyes. (f) Knockdown of Tctp results in small and rough eye phenotype. (g,h) Knockdown of both Tctp and $14-3-3 \varepsilon$ $(\mathbf{g})$ or $14-3-3 \zeta(\mathbf{h})$ strongly enhances the eye phenotype caused by Tctp RNAi alone. (i,j) One copy of $14-3-3 \varepsilon^{\text {j2B10 }}(\mathbf{i})$ or $14-3-3 \zeta 07103(\mathbf{j})$ enhances the Tctp RNAi phenotype. (k-p) Genetic interaction between Tctp and 14-3-3 in wing. (k) Control wing with one copy of nub-Gal4 is normal. (I) Knockdown of

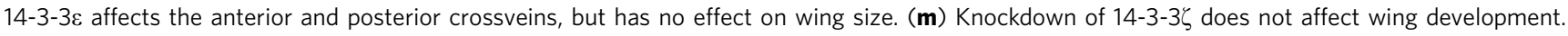

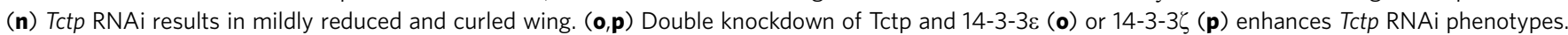
RNAi knockdown was induced by ey-Gal4 (a-c,f-j) and nub-Gal4 (k-p). Scale bar, $200 \mu \mathrm{m}(\mathbf{a}-\mathbf{j}) ; 400 \mu \mathrm{m}(\mathbf{k}-\mathbf{p})$. 

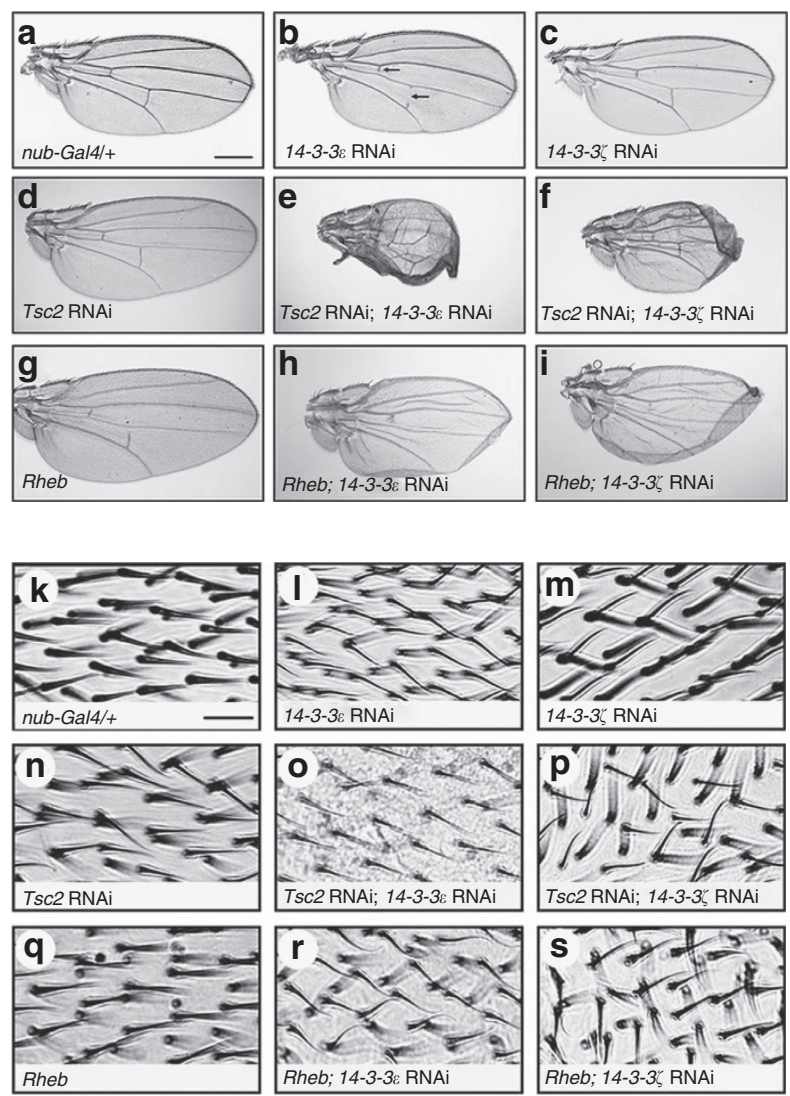
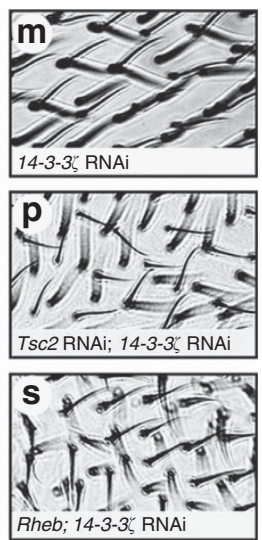
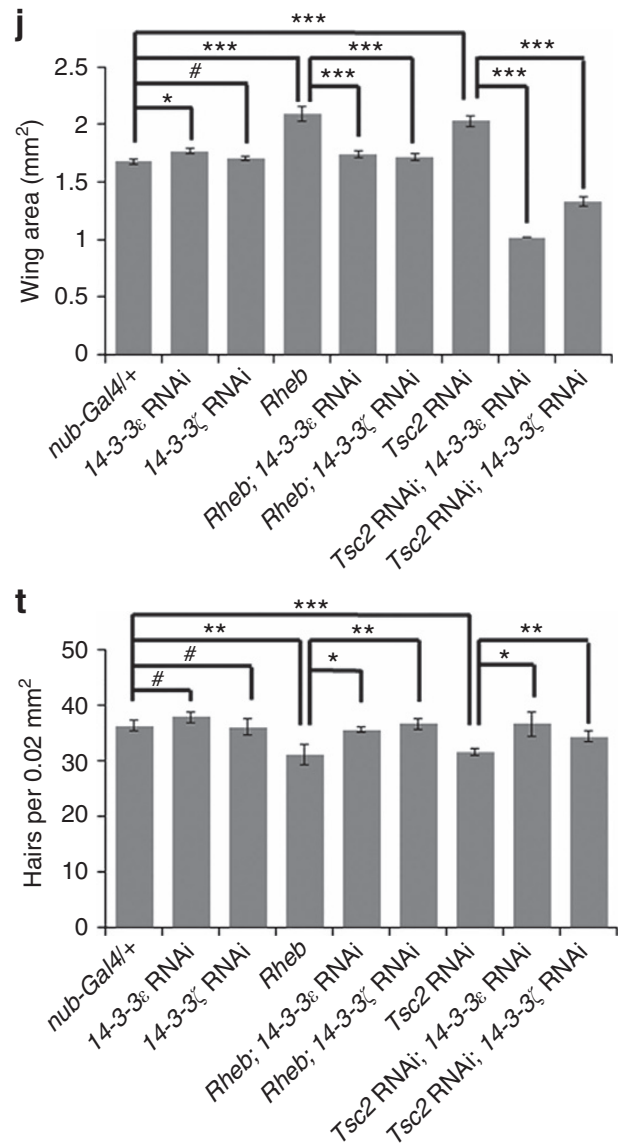

Figure 2 | Genetic interaction of 14-3-3 with Tsc2 and Rheb in the wing. (a-f) 14-3-3 shows genetic interaction with Tsc2. (a) Control wing with one copy

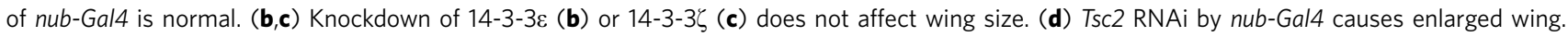
(e,f) Reduction of 14-3-3 levels in Tsc2 RNAi background results in inhibition of overgrowth and shape changes in wing. (g-i) Genetic interaction between

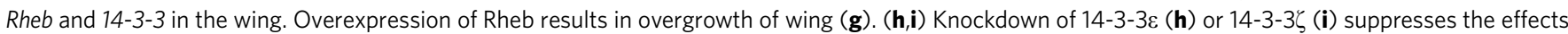
of Rheb overexpression. RNAi or overexpression was induced by nub-Gal4 (a-i). Scale bar, $400 \mu \mathrm{m}(\mathbf{a}-\mathbf{i})$. (j) Quantification of wing sizes shown in a-i. Knockdown of one isoform of 14-3-3 does not affect wing size compared with the control (nub-Gal4/+). Knockdown of Tsc2 or overexpression of Rheb increases the wing size about 20\% compared with wild-type. Knockdown of one 14-3-3 isoform suppresses overgrowth phenotype caused by Rheb overexpression. Knockdown of one 14-3-3 isoform and Tsc2 results in about 30\% reduction of wing size compared with the wild-type size. Error bars are s.d. $n=10$. ${ }^{\star} P<0.01$. ${ }^{\star \star}{ }^{\star} P<0.0001$. ${ }^{\#} P>0.05$ (t-test). (k-s) High magnification images of flat areas of adult wings with indicated genotypes. Scale bar,

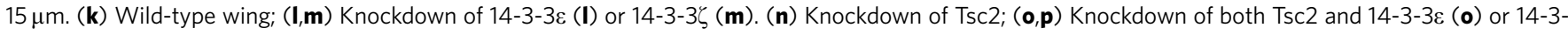

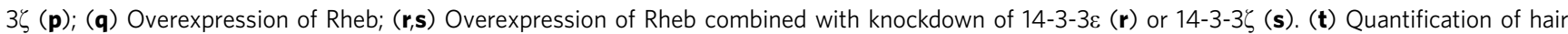
numbers within the same area of wings $\left(0.02 \mathrm{~mm}^{2}\right)$. Knockdown of one isoform of 14-3-3 does not affect the hair number. Knockdown of Tsc2 or overexpression of Rheb causes about 20\% reduction in the wing hair number compared with wild-type. Knockdown of one isoform of 14-3-3 combined with Tsc2 RNAi or Rheb overexpression results in similar wing hair numbers as wild-type. Error bars are s.d. $n=10 .{ }^{\star} P<0.01 .{ }^{\star \star} P<0.001$. ${ }^{\star \star \star} P<0.0001$.

${ }^{\#} P>0.05$ (t-test).

14-3-3s are required for Tctp-Rheb interaction and growth. The data that 14-3-3s bind to both Tctp and Rheb led to a question if 14-3-3s are required for the interaction between Tctp and Rheb to form a complex. To test this hypothesis, we knocked down $14-3-3 \mathrm{~s}$ in S2 cell culture, and checked whether reduced 14-3-3s might affect the interaction between Tctp and Rheb. 14-3-3 proteins were depleted in day 6th after treating S2 cell with 14-3-3s RNAi (Fig. 5a). As shown in a previous study ${ }^{29}$, knockdown of one isoform of 14-3-3s resulted in upregulation of the other isoform to compensate the loss of the targeted isoform (Fig. 5a). When $14-3-3 \varepsilon$ or $14-3-3 \zeta$ was depleted, there was no consistent change in the interaction between Tctp and Rheb, as assayed by co-IP. However, double knockdown of both 14-3-3 isoforms resulted in near complete loss of the binding between Tctp and Rheb (Fig. 5b). Levels of Tctp and Rheb were not altered by depleting 14-3-3s, indicating that the loss of Tctp-Rheb binding was not due to reduction of these proteins (Fig. 5a).
As shown above, single knockdown or loss-of-function mutation of either one of 14-3-3 genes did not affect organ growth. Since depletion of both 14-3-3 forms results in the disruption of physical interaction between Tctp and Rheb (Fig. 5b), we examined the effects of depleting both 14-3-3 isoforms in developing organs. In contrast to single knockdown that did not noticeably affect development (Fig. $5 \mathrm{c}-\mathrm{e}$ ), double knockdown of these two 14-3-3 genes resulted in pupal lethality. Removal of the pupal case from developing pupae revealed that double knockdown of both 14-3-3 genes causes loss of nearly entire eye and head tissues (Fig. 5f). Furthermore, dissection of third instar larvae showed massive disruption of eye-antenna discs, consistent with the loss of eye and head in pupal tissues (Fig. 5j). In contrast, single knockdown of either 14-3-3 isoform did not cause obvious defects in eye growth (Fig. 5h,i). Single RNAi knockdown in wing disc also did not affect cell proliferation or cell death marked by staining for 

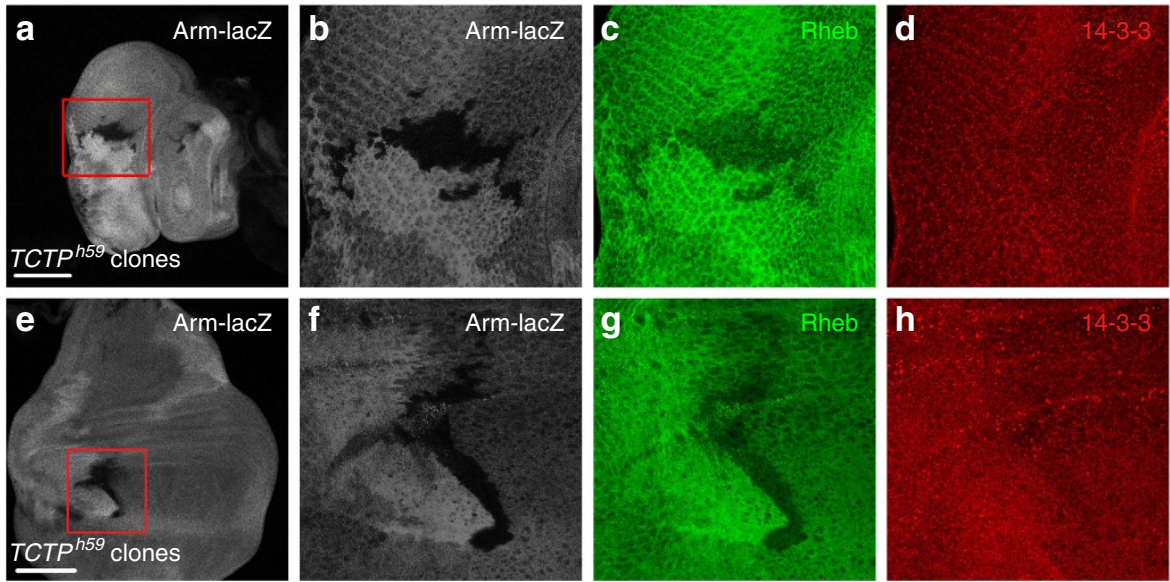

Figure 3 | Effects of Tctp mutation on Rheb and 14-3-3s. (a-d) Clones of Tctp ${ }^{h 59}$ null mutant cells in eye disc. (e-h) Clones of Tctph59 null mutant cells in wing disc. These clones are marked by the absence of Arm-lacZ. In both organs, Tctp mutant clones show reduction of Rheb (c,g) but not 14-3-3s (d,h). Red rectangles in $\mathbf{a}$ and $\mathbf{e}$ indicate the enlarged regions shown in $\mathbf{b}-\mathbf{d}$ and $\mathbf{f}-\mathbf{h}$ respectively. Scale bar, $100 \mu \mathrm{m}$ (a-h).

a

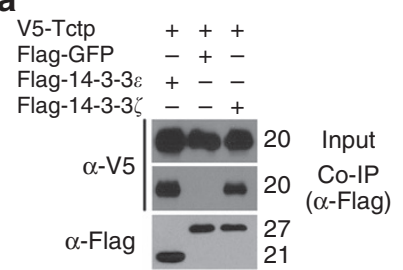

b

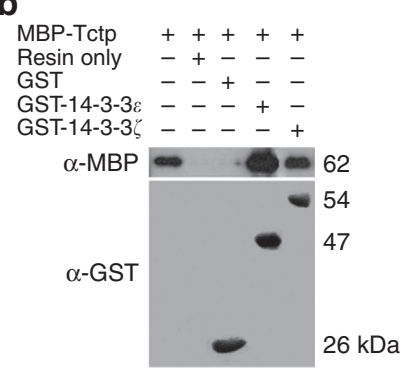

C

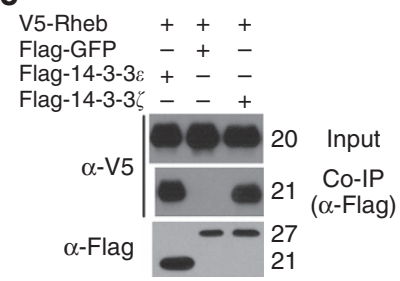

d

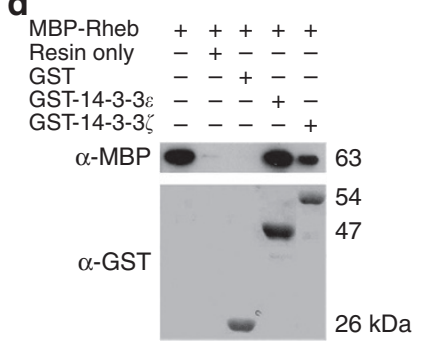

Figure 4 | Tctp and Rheb physically interact with 14-3-3s.

(a) Co-immunoprecipitation of $14-3-3 \mathrm{~s}$ and Tctp. S2 cells were transfected with indicated genes. First western blot (WB) shows 5\% input of V5-Tctp. Second blot shows V5-Tctp co-immunoprecipitated by Flag-14-3-3 $\varepsilon$ and

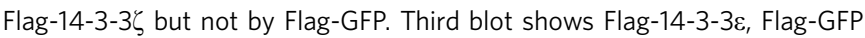
and Flag-14-3-3ל immunoprecipitated with anti-Flag. (b) Direct binding

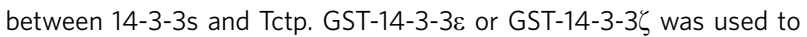
pull-down MBP-Tctp as indicated. First blot shows MBP-Tctp proteins stained by anti-MBP. First lane indicates $5 \%$ input of MBP-Tctp used for

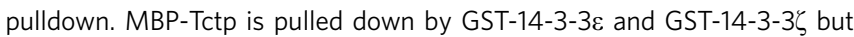
not by GST. Second blot shows GST and GST-fusion proteins stained by anti-GST. (c) Co-immunoprecipitation of 14-3-3s and Rheb. First blot shows $5 \%$ input of V5-Rheb. Second blot shows V5-Rheb co-immunoprecipitated

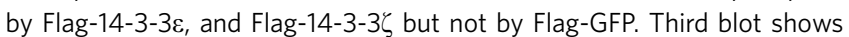

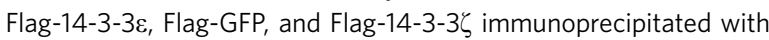
anti-Flag. (d) Direct binding between 14-3-3s and Rheb. GST-14-3-3e or GST-14-3-3 $\zeta$ was used to pull-down MBP-Rheb as indicated. First blot shows MBP-Rheb stained by anti-MBP. First lane indicates $5 \%$ input of MBP-Rheb used for pulldown. MBP-Rheb is pulled down by GST-14-3-3E and GST-14-3-3ל but not by GST. Second blot shows GST and GST-fusion proteins stained by anti-GST.

phospho-Histone 3 ( $\mathrm{PH} 3$; Fig. 5k-m) or activated Caspase 3 (Cas-3) (Fig. 5o-q), respectively. However, knockdown of both 14-3-3 isoforms strongly reduced $\mathrm{PH} 3$ staining (Fig. 5n) while increasing activated Caspase 3 (Fig. 5r). These results indicate that loss of both 14-3-3 isoforms critically impairs organ development by affecting cell proliferation and cell survival.

Silencing 14-3-3s affects downstream targets of Tor. Rheb activates Tor kinase, which leads to cell growth and proliferation through S6 kinase (S6k), Thor and CycE, respectively ${ }^{7,39}$. It has been shown that S6k phosphorylation is greatly reduced by Tctp silencing ${ }^{8}$. On the basis of genetic and physical interaction of 14-3-3s with Tctp and Rheb, we tested whether 14-3-3 knockdown can affect Tor activity by checking the levels of phosphorylated S6k and Thor in S2 cells. Either single or double knockdown of $14-3-3$ s resulted in similar reduction of pS6k detected by anti-pS6k (Thr 398) antibody, while there was little change in the total level of S6k protein. Phosphorylated Thor was strongly reduced by either single or double knockdown of 14-3-3s (Fig. 6a). Double knockdown of 14-3-3s resulted in some reduction of Thor protein levels while single knockdown did not.

To check the effects of 14-3-3 mutations on CycE, we generated 14-3-3E mutant clones in eye discs. These clones showed reduced

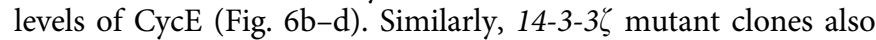
resulted in a reduction of $\mathrm{CycE}$ level (Fig. 6e-g). However, such partial reduction of $\mathrm{CycE}$ by loss of one 14-3-3 isoform had little effect on the growth of mutant clones in the eye disc (Supplementary Fig. 1a-c,g-i), although reduced 14-3-3 causes abnormal differentiation of photoreceptor cells ${ }^{30,31}$.

Defects by Tctp and 14-3-3 knockdown are suppressed by CycE. Because both Tctp and 14-3-3s affect Tor signalling, we tested whether double knockdown of Tctp and 14-3-3 can be suppressed by S6k and CycE. Growth defects from double knockdown of Tctp and 14-3-3 could not be suppressed by overexpressing S6k alone (Supplementary Fig. 4). Next, we tested the effects of overexpressing CycE. Single knockdown of either form of 14-3-3 strongly enhanced the effects of Tctp RNAi, as shown earlier (Fig. 1; Supplementary Fig. 2). Overexpression of CycE restored the small eye phenotype caused by knockdown of Tctp and one of $14-3-3 \mathrm{~s}$ in $\sim 80 \%$ of flies examined (Fig. $6 \mathrm{k}-\mathrm{n}$ ), while $\mathrm{CycE}$ overexpression in the wild-type background did not increase the eye size (Fig. 6i). The small-wing phenotype resulting from double knockdown of Tctp and one of 14-3-3 isoforms was 


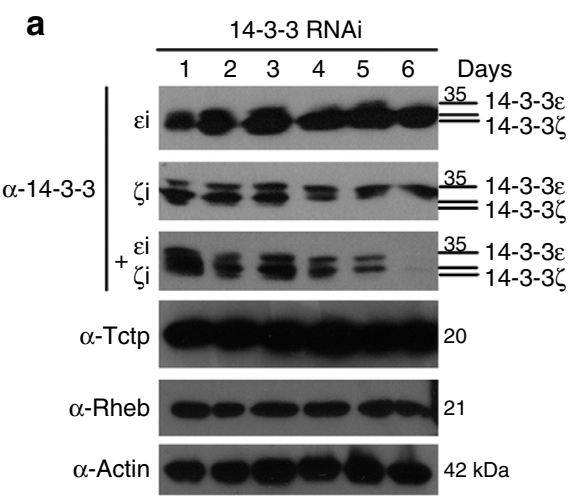

b
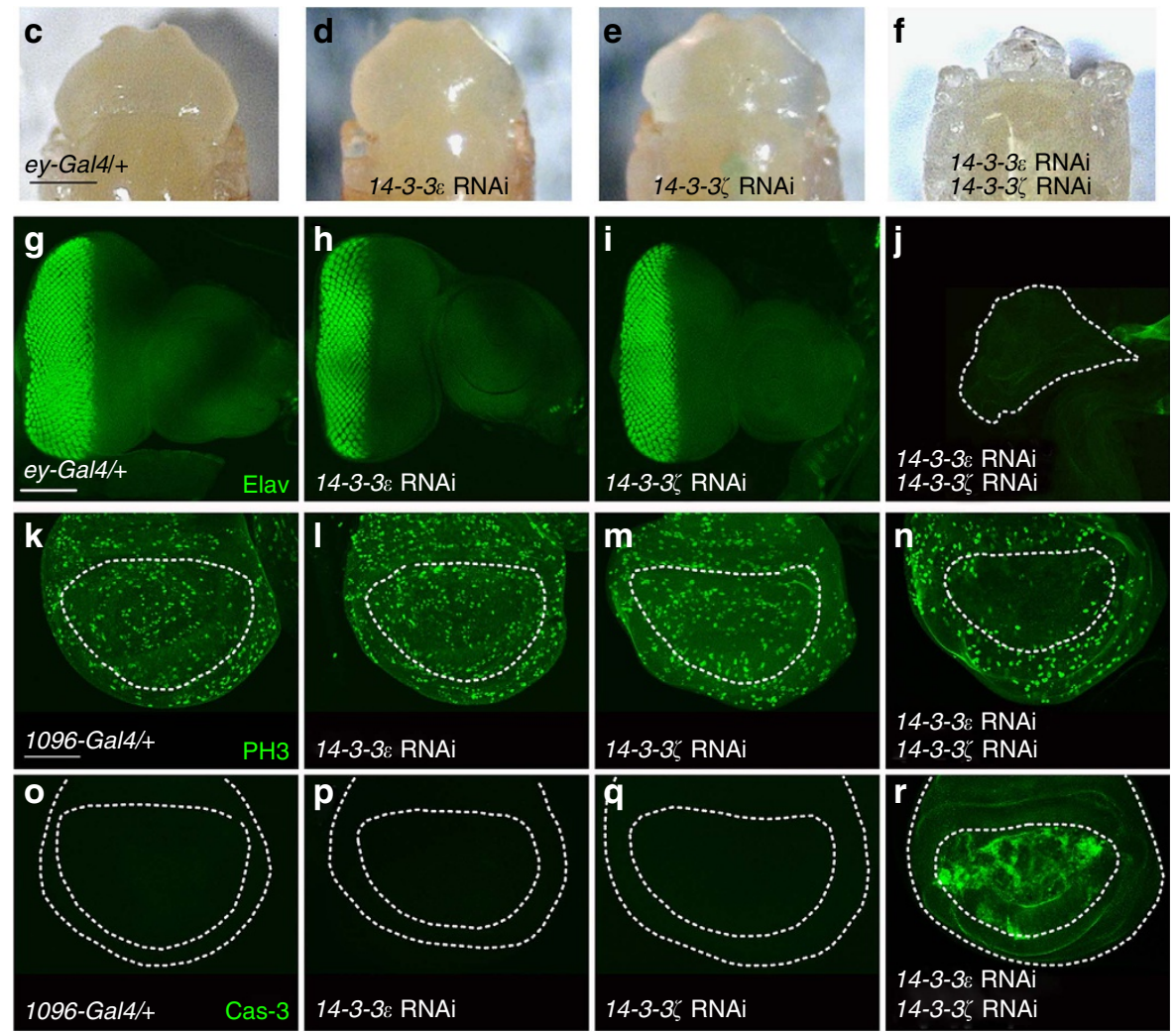

Figure 5 | 14-3-3s are required for the interaction between Tctp and Rheb and organ development. (a) Knockdown of 14-3-3s in S2 cells shows depletion of $14-3-3$ s levels after 6 days of RNAi treatment. $\varepsilon i, \zeta i$ and $\varepsilon i+\zeta i$ indicate RNAi for $14-3-3 \varepsilon, 14-3-3 \zeta$ and both isoforms, respectively. Levels of Tctp and Rheb were not noticeably affected by 14-3-3 depletion. The treatment performed to detect Tctp, Rheb, and actin level was double knockdown of both isoforms of 14-3-3. (b) 14-3-3 depletion affects the interaction between V5-Tctp and V5myc-Rheb after 6 days of RNAi treatment. Anti-Myc antibody was used for IP. Tctp was detected by anti-Tctp antibody. Depletion of both isoforms abolished the Tctp-Rheb interaction. (c-j) Double knockdown of

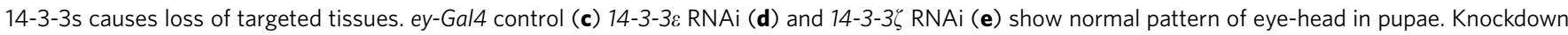
of both 14-3-3 isoforms causes pupal lethality with loss of eye and head (f). ( $\mathbf{g}-\mathbf{j}$ ) Pattern of Elav staining in larval eye discs from indicated genotypes. Knockdown of $14-3-3 \varepsilon$ or $14-3-3 \zeta$ has no obvious effect on eye disc development (h,i). Knockdown of both isoforms results in small disc (the area marked with dotted line) with no retinal differentiation (j). (k-n) Double knockdown of 14-3-3s results in reduced proliferation. MS1096-Gal4 control (k) $14-3-3 \varepsilon$

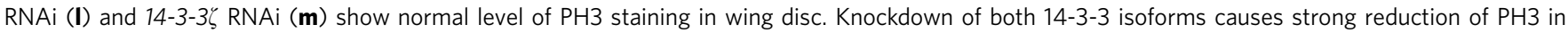
the wing pouch (circled area) (n). (o-r) Caspase activation marked by anti-Caspase 3 (Cas-3) staining is not detected in MS1096-Gal4 control (o) 14-3-38 RNAi (p) and 14-3-3ל RNAi (q). Double knockdown of both 14-3-3 isoforms results in strong activation of Cas-3 (r). Scale bar, $300 \mu \mathrm{m}(\mathbf{c}-\mathbf{f}) ; 100 \mu \mathrm{m}(\mathbf{g}-\mathbf{j})$; $100 \mu \mathrm{m}(\mathbf{k}-\mathbf{r})$.

also partially rescued by CycE in nearly $100 \%$ flies examined (Fig. 6o-r).

We also examined whether $\mathrm{CycE}$ can suppress the effects of depleting both forms of 14-3-3 on wing development. Double knockdown of 14-3-3 isoforms in the wing pouch by MS1096-Gal4 led to more than 90\% pupal lethality and severe reduction of wing tissues in the surviving adult flies (Supplementary Fig. 5c). CycE overexpression in the wild-type background did not affect the wing size or survival (Supplementary Fig. 5b). In contrast, CycE overexpression in the 14-3-3 double knockdown condition suppressed the wing growth defect in $>80 \%$ of the flies examined (Supplementary Fig. $5 \mathrm{~d}-\mathrm{g}$ ), as well as pupal lethality ( $90 \%$ survival). These results suggest that reduced 14-3-3s synergize with partial loss of Tctp to affect the Tctp-Rheb interaction and Tor signalling, leading to a reduction of the CycE level. 
a

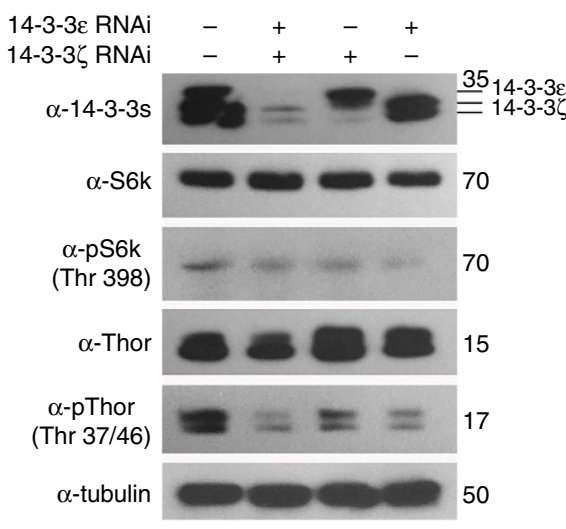

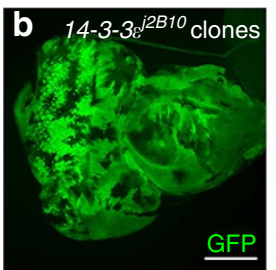

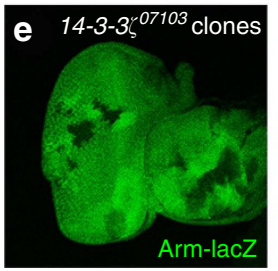

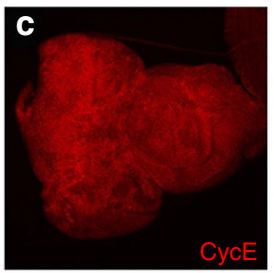
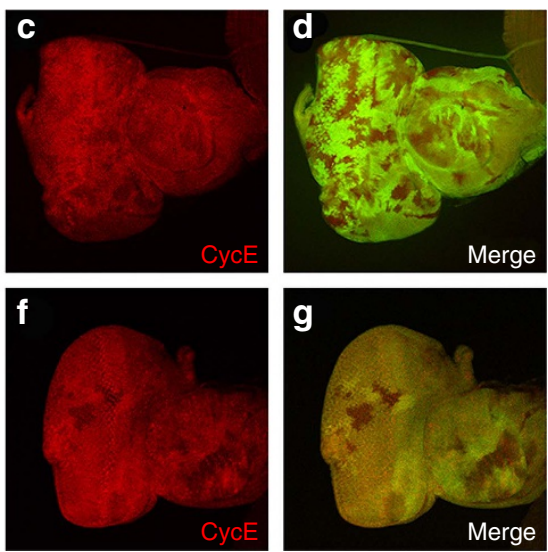
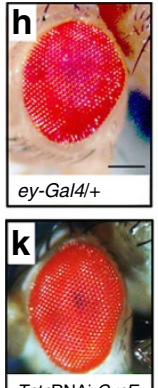

TctpRNAi; CycE
14-3-3 RNAi(S)
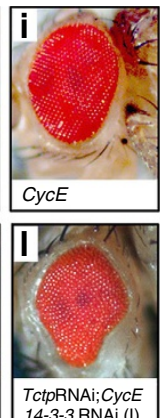

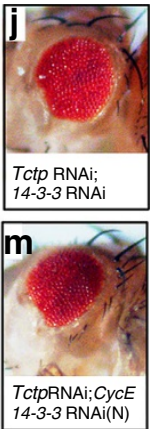

n

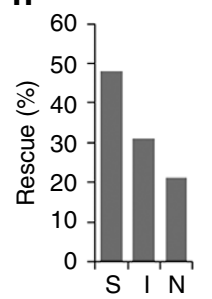

S: Strong rescue

I: Intermediate rescue

$\mathrm{N}$ : No rescue
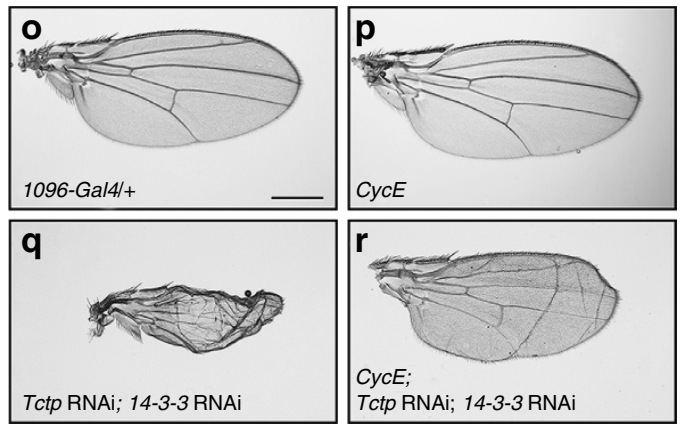

Figure 6 | Effects of 14-3-3 RNAi on Tor targets and the suppression by CycE. (a) Effects of 14-3-3 RNAi on S6k and Thor. Either one of 14-3-3 isoforms or both 14-3-3s were knocked down in S2 cells. Single or double knockdown of 14-3-3s reduced the level of pS6k (Thr 398) but does not affect the level of S6k protein. Knockdown of both 14-3-3s, but not one isoform, causes slight reduction of Thor. In contrast, pThor (Thr 37/46) is strongly reduced by single or double knockdown of $14-3-3$ s. (b-d) Loss of $14-3-3$ s results in reduction of CycE level. (b) $14-3-3 \varepsilon$ mutant clones induced by ey-flp are marked by the absence of GFP. Mutant clones show strong reduction of CycE (c). (e-g) 14-3-3 $\zeta$ mutant clones induced by hs-flp are marked by the absence of Arm-lacZ. CycE is reduced in mutant clones (f). (h-m) Effects of CycE in eye. Control with one copy of ey-Gal4 (h) or overexpression of CycE (i) shows normal eye. (j) Double knockdown of Tctp and 14-3-3 results in small and rough eye phenotype ( $<40 \%$ of wild-type eye size). (k-m) Overexpression of CycE rescues the small-eye phenotype resulting from double knockdown of Tctp and 14-3-3. The rescue is varied, ranging from strong (S, 81-100\% of wild-type size) (k), intermediate (I, 41-80\%) (I) to no rescue (N,40\% or lower) (m). (n) Quantification of rescue shown in k-m. More than 60 flies with the genotype of ey > Tctp RNAi/CycE GFP; 14-3-3 RNAi/ + were scored. (o-r) CycE suppresses the wing phenotypes of double knockdown of Tctp and 14-3-3. Control with one copy of MS1096-Gal4 (o) or CycE overexpression (p) does not affect wing development. (q) Knockdown of both Tctp and 14-3-3 results in small and wrinkled wing. (r) Overexpression of CycE partially rescues the double-knockdown phenotype in 100\% of tested flies. Scale bar, $100 \mu \mathrm{m}$ (b-g); $200 \mu \mathrm{m}(\mathbf{h}-\mathbf{m}) ; 400 \mu \mathrm{m}(\mathbf{o}-\mathbf{r})$.

\section{Discussion}

Rheb plays a key role in Tor signalling by activating Tor kinase. Thus, how the Rheb function is regulated is an important issue. In this study, we have shown that 14-3-3 proteins play important roles in organ growth by affecting Tctp and Rheb for positive regulation of Tor signalling. Functional analysis of 14-3-3 genes in vivo has been limited due to their redundant roles. A clue for the role of 14-3-3s in Tor signalling in vivo was obtained from our finding of strong genetic interaction between Tctp and 14-3-3.

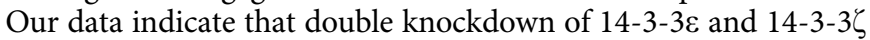
isoforms causes severe defects in organ growth, whereas silencing of either one of these isoforms has little effect. Interestingly, knockdown of $14-3-3 \varepsilon$ or $14-3-3 \zeta$ together with Tctp RNAi results in synergistic growth defects. 14-3-3 genes also show strong genetic interaction with Rheb and Tsc2 in which knockdown of one 14-3-3 isoform suppresses the wing overgrowth caused by Rheb overexpression or Tsc2 silencing. Knockdown of either $14-3-3 \varepsilon$ or $14-3-3 \zeta$ has little effect on wing development. But together with Rheb overexpression, it results in smaller and curled-down wings in comparison with Rheb-overexpressed wing. Similarly, 14-3-3 RNAi did not simply suppress the overgrown wing phenotype of Tsc2 RNAi to normal wing size. Instead, wings with knockdown of both 14-3-3 and Tsc2 are much smaller than the normal size of 14-3-3 RNAi wings. Currently, it is unknown how 14-3-3 RNAi negatively affects wing tissues under Rheb overexpression or Tsc2 knockdown conditions while 14-3-3 RNAi alone does not affect wing growth in the wild-type condition. It has been reported that tissues from $T s c 1$ or Tsc2 mutant patients show abnormal expression in a number of genes ${ }^{40}$. Therefore, it is possible that wing tissues with Rheb overexpression or Tsc2 RNAi may have alterations in many intracellular events. Under these conditions, such tissues might become more sensitive to a reduction of 14-3-3 than wild-type tissues, resulting in abnormal or reduced wings. Alternatively, activated Tor signalling might affect other linked signalling pathways to induce cell death under certain conditions such as reduced 14-3-3. Some oncogenic genetic changes are known to promote apoptosis ${ }^{41}$. In case of Tor signalling, Tsc-deficient cells can constitutively activate Rheb-Tor signalling, which inhibits Akt signalling to promote cell death ${ }^{42}$. Therefore, it is possible that Tsc2 depletion or Rheb overexpression might lead to downregulation of other 14-3-3-dependent growth signalling pathways, thus resulting in wing growth defects when 14-3-3 levels are compromised. Identification of the mechanism underlying the synergistic interaction between 14-3-3 and Rheb/Tsc2 needs further studies. 
In addition to genetic interaction, 14-3-3, Tctp, and Rheb physically interact with each other. Double knockdown of both 14-3-3 isoforms results in reduction of Tor downstream components such as phosphorylated S6k, phosphorylated Thor and $\mathrm{CycE}$ (Fig. 6a,b). In S2 culture cells, 14-3-3 RNAi did not affect the level of total S6k but it reduced the level of pS6k even by single knockdown of $14-3-3 \varepsilon$ or $14-3-3 \zeta$. Phosphorylated Thor was strongly reduced by knockdown of a single 14-3-3 isoform (Fig. 6a). On the contrary, single knockdown of 14-3-3e did not strongly affect the interaction between Tctp and Rheb (Fig. 5b). These data seem to suggest that 14-3-3s may not affect TORC1 activity by modulating Tctp-Rheb binding. However, it is worth noting that 14-3-3s may also affect other Tor components such as Tsc2. Different 14-3-3 isoforms are known to inhibit Tsc2 in mammalian cells by binding to Tsc2 phosphorylated by $\mathrm{Akt}^{43}$. Therefore, although single knockdown of 14-3-3 may not strongly affect Tctp-Rheb interaction, depletion of a 14-3-3 isoform could elevate Tsc2 function to inhibit Rheb activity, thus reducing pS6k and pThor as shown in Fig. 6a. Our data for genetic interactions between 14-3-3 and Tctp (or Rheb) support the role of 14-3-3 affecting Tor signalling by interacting with Tctp and Rheb, although we do not exclude the possibility that 14-3-3s may also affect Tor signalling independent of Tctp-Rheb interaction.

An intriguing question is how single knockdown of 14-3-3 affects Tor signalling in S2 cells but not in animal tissues like eye/wing discs. Such difference might be due to cell- or tissue-specific function of Tor signalling ${ }^{44}$. For example, Tsc2 phosphorylation by Akt increases Tor signalling in mammalian cells. Although Drosophila Tsc2 is also phosphorylated by Akt in cultured S2 cells, Tsc2 phosphorylation does not play a critical role during normal development ${ }^{45}$. Thus, signalling events can vary between culture cells and intact organisms or even between different culture cell types. We noted that double knockdown of 14-3-3s fails to completely eliminate pS6k and pThor. Low levels of residual $14-3-3$ (either $14-3-3 \varepsilon$ or $14-3-3 \zeta$ ) after RNAi treatment might be sufficient to keep some of TORC1 activity. It is also possible that Tor might be activated in part by Rheb-independent mechanisms in S2 cells as reported in mammalian cells ${ }^{46}$.

Mammalian Tctp proteins are known to be phosphorylated at several sites by Polo-like kinase 1 (ref. 47) and insulin signalling ${ }^{48}$. Mammalian Rheb is phosphorylated at Ser-130 by p38 regulated/activated protein kinase (Prak) ${ }^{49}$. Although Drosophila Tctp and Rheb have conserved Ser or Thr residues at the similar positions, no experimental evidence for phosphorylation has been reported. It seems that these serine or threonine sites do not belong to two conserved 14-3-3 binding motifs $\mathrm{R}[\mathrm{S} / \mathrm{Ar}] \mathrm{XpSXP}$ and $\mathrm{RX}[\mathrm{Ar} / \mathrm{S}] \mathrm{XpSXP}$, where $\mathrm{Ar}$ is an aromatic amino acid. However, some 14-3-3 binding ligands have sequences diverged from these motifs or do not require phosphorylation for binding ${ }^{50}$. Therefore, it remains to be determined whether 14-3-3 binding to Tctp is mediated through phosphorylation.

Importantly, our data show that the binding between Tctp and Rheb is almost completely disrupted by knockdown of both 14-3-3 isoforms but not by a single knockdown. This suggests that 14-3-3 isoforms might play a redundant role to promote direct interaction between Tctp and Rheb. Since 14-3-3s bind directly to both Tctp and Rheb, Tctp-Rheb binding might be promoted, possibly by using the known dimerization of 14-3-3 proteins ${ }^{29,50}$. For such interactions to occur, these three proteins must be present in a stoichiometric ratio to form a proper complex. In our co-IP assays in S2 cells, the levels of endogenous 14-3-3 proteins were similar to the levels of exogenous Tctp and Rheb (Supplementary Fig. 6), suggesting that 14-3-3s, Tctp and Rheb are present in a stoichiometric ratio to form a direct complex. However, it is also possible that 14-3-3s might interact separately with Tctp and Rheb to promote the binding between Tctp and Rheb without forming a direct complex coupled by 14-3-3 proteins. Further studies are necessary to identify the precise mechanism of molecular interactions among these proteins.

Our study reveals a novel function of 14-3-3 for promoting the Tctp-Rheb interaction at the critical step of Tor activation. 14-3-3 proteins are also known to regulate Tor signalling by interacting with Tsc2, Pras40 and Raptor ${ }^{20-24}$, although in vivo functions of these interactions have not been studied in Drosophila. Thus, 14-3-3 isoforms may be involved in more steps than those known so far, both up and/or downstream of Tor kinase. It would be important to study how such diverse 14-3-3 interactions with multiple Tor components contribute to generate signalling outputs for cell growth and proliferation. Nonetheless, the new function of 14-3-3 isoforms for the interaction between Tctp and Rheb is crucial for organ growth in Drosophila. It would be interesting to see whether the function of 14-3-3s shown in this study is conserved in vertebrates and whether loss of a functionally redundant human 14-3-3 isoform can synergize with partial defects in Tor signalling components, leading to more severe pathological conditions.

\section{Methods}

Fly genetics. 14-3-3 RNAi fly lines were obtained from the Vienna Drosophila Resource Center (VDRC) and the National Institute of Genetics (NIG). 14-3-38

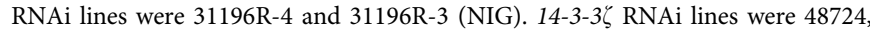
48725,104496 (VDRC) and 17870R-2 (NIG). 14-3-3e loss-of-function mutant $\operatorname{strain}^{30}\left(y^{d 2} w^{1118}\right.$ ey-flp; GMR-lacZ; FRT82B 14-3-3e $\varepsilon^{2 B 10} / T M 6$ Tb) was from the Drosophila Genomics Resource Center (DGRC). 14-3-3 $\zeta^{07103}$ P-insertion lethal mutant $\operatorname{strain}^{31}\left(w ;\right.$ FRT42D 14-3-3 $\left.\zeta^{07103} / C y O\right)$ was from the Bloomington Drosophila Stock Center. Tctp RNAi and mutant line FRT82B Tctp ${ }^{h 59} / T M 6 T b$ were as described ${ }^{8}$. Two other Tctp RNAi lines from VDRC were v45531 and v45532. Flies were grown at room temperature, unless stated otherwise.

For double knockdown of 14-3-3s, MS1096-Gal4 and UAS-14-3-3e RNAi were recombined on the first chromosome. MS1096 > 14-3-3e RNAi recombinant females were crossed with male UAS-14-3-3 $\zeta$ RNAi.

For double knockdown of Tctp and 14-3-3, ey>Tctp $\mathrm{RNAi} / \mathrm{CyO}$ recombinants

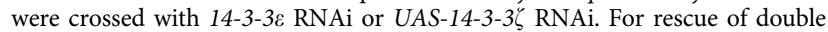
knockdown of 14-3-3s by CycE, MS1094>14-3-3e RNAi recombinant female flies were crossed with $U A S-C y c E$ GFP on the second chromosome. Progeny from

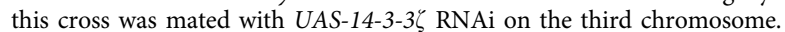
Larvae showing green fluorescent protein (GFP) expression with the genotype $M S 1096>14-3-3 \varepsilon \mathrm{RNAi} /+$; $C y c E \mathrm{GFP} /+$; $14-3-3 \zeta \mathrm{RNAi} /+$ were selected to check phenotypes.

For rescue of double knockdown of Tctp and 14-3-3 by CycE, ey $>$ Tctp RNAi/CyO; $14-3-3 \varepsilon$ RNAi/TM6 Tb or $e y>$ Tctp RNAi/CyO; 14-3-3 6 RNAi/TM6 Tb were selected based on eye phenotype. These recombinant flies were crossed with $U A S-C y c E$ GFP, and the progeny with GFP expression and without TM6 Tb balancer was kept for further analysis.

Generation of mosaic clones in eye and wing discs. Mutant clones were generated by FLP-FRT method ${ }^{51}$. Clones of 14-3-3e mutation in eye discs were made using $14-3-3 \varepsilon^{j 2 B 10}$ mutant fly line ( $y^{d 2} w^{1118}$ ey-flp; GMR-lacZ; FRT82B 14-3-3 $\varepsilon^{2 B 10} / T M 6 T b$ ) from DGRC. 14-3-3e mutant flies were crossed with FRT82B $u b i-G F P / T M 3 S b$ fly (Bloomington). Eye discs from third instar larval progeny were dissected and immunostained with antibodies indicated in each figure. Mutant clones were identified by the absence of GFP.

To generate $14-3-3 \varepsilon$ mutant clones in wing discs, we crossed $h s-f l p ;$ FRT82B ubi-GFP/TM3 Sb with $y^{d 2} w^{111}$ ey-flp; GMR-lacZ; FRT82B 14-3-3e 2 iB10/TM6 Tb. First instar larvae from this cross were heat shocked at $37^{\circ} \mathrm{C}$ for $1 \mathrm{~h}$ and incubated at $25^{\circ} \mathrm{C}$ until third instar stage. Wing discs were dissected for immunostaining. Mutant clones were identified by the absence of GFP staining. To generate 14-3-3 $\zeta$ mutant clones, $w$; FRT42D 14-3-3 $\zeta^{07103} / C y O$ flies were crossed with $h s-f l p ; F R T 42 D$ arm-lacZ. First instar larvae were heat shocked as described above, and eye and wing discs from third instar larvae were dissected for immunostaining. Mutant clones were identified by the absence of LacZ staining. Tctp mutant clones were generated by crossing $h s$-flp; FRT82B arm-lacZ with $w$; FRT82B Tctp ${ }^{h 59} / T M 6 B$ Tb. First instar larvae were heat shocked as described above, and imaginal discs from third instar larvae were dissected for immunostaining.

Generation of anti-Rheb antibody. Five-hundred forty-nine-base pair full-length Rheb complementary DNA (cDNA) including stop codon was cloned to pMAL-c2 
vector. MBP-Rheb fusion protein was purified from bacteria and used for generating rat anti-Rheb antibody from Abclon. Anti-Rheb antibody was used at 1:500 for western blot and 1:100 for immunocytochemistry as described below.

Immunocytochemistry. Third instar eye and wing imaginal discs were dissected in $1 \times$ PBS, fixed in PLP $(2 \%$ paraformaldehyde, $0.01 \mathrm{M}$ periodate, $0.075 \mathrm{M}$ lysine and $0.035 \mathrm{M}$ phosphate buffer) for $30 \mathrm{~min}$, and incubated with primary antibodies at $4{ }^{\circ} \mathrm{C}$ overnight. After washing, imaginal discs were incubated with secondary antibodies for $2 \mathrm{~h}$ at room temperature. First, antibodies were used as following: rat anti-Rheb at 1:100, rabbit anti-Tctp ${ }^{8}$ at 1:200, rabbit anti-14-3-3 (Invitrogen 51-0700) at 1:200, mouse anti- $\beta$-gal (DSHB 40-1a) at 1:50, rat anti-Elav (DSHB $7 \mathrm{E} 8 \mathrm{~A} 10$ ) at $1: 100$, rabbit anti-PH3 (Milipore) at 1:200, rabbit anti-Cas-3 (Cell Signaling 9661) at 1:300, sheep anti-GFP (Ab-direct Serotec 4745-1051) at 1:100, rabbit anti-CycE (Santa Cruz) at 1:100 and rabbit anti-Dlg (from Dr Kyung-Ok Cho) at 1:500. Secondary antibodies conjugated with Cy3, Cy5 or FITC (Molecular Probes) were: anti-rabbit Cy3, anti-rat FITC, anti-mouse Cy5, and anti-sheep FITC. Mounting solution was Vectashield with 4',6-diamidino-2-phenylindole (Vector Laboratories H-1200). Fluorescent images were acquired using Carl Zeiss confocal microscope.

In vitro GST pull-down assays. $14-3-3 \varepsilon$ cDNA (LD27892) and $14-3-3 \zeta$ cDNA

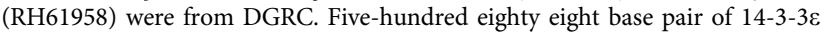
coding sequence (including stop codon) was amplified by PCR reaction and cloned

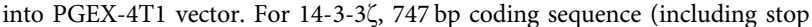
codon) was cloned into PGEX-4T1 vector. Tctp- and Rheb-coding sequences were cloned into pMAL-c2 vector. Fusion tagged proteins were expressed in BL21 Escherichia coli. For GST pull-down assay, $20 \mu \mathrm{g}$ of each MBP and GST-tagged protein were added to $500 \mu \mathrm{l}$ pull-down buffer $(20 \mathrm{mM}$ Tris $\mathrm{pH} 7.5,150 \mathrm{mM} \mathrm{NaCl}$, $0.5 \mathrm{mM}$ EDTA, $10 \%$ glycerol, $0.1 \%$ Triton X-100, $1 \mathrm{mM}$ dithiothreitol, $1 \mathrm{mM}$ $\mathrm{Na}_{3} \mathrm{VO}_{4}, 10 \mathrm{mM} \mathrm{NaF}$ and a protease inhibitor cocktail (Roche 11873580 001)), followed by incubation overnight at $4{ }^{\circ} \mathrm{C}$ with Glutathione Excellose resin (Bioprogen). After 3 times of washing with cold pull-down buffer, $5 \times$ SDS loading buffer was added and samples were boiled at $94^{\circ} \mathrm{C}$ for $5 \mathrm{~min}$, then centrifuged at 12,000 r.p.m for $5 \mathrm{~min}$ and loaded. For immunostaining of western blots, rabbit anti-MBP antibody (Santa Cruz) and secondary anti-rabbit antibody (Jackson) were used at 1:5,000 and 1:10,000

Co-immunoprecipitation assays. Coding sequences of 14-3-3s were cloned into Flag-tagged pRmHa-3 vector (DGRC). Tctp- and Rheb-coding sequences were cloned into pAC-V5HisA vector (Invitrogen). For co-IP between Tctp and Rheb, Rheb-coding sequence was cloned into pAC-V5mycHisA vector (Invitrogen). Co-IP assays were carried out using a standard protocol ${ }^{52}$ with minor modifications. DNA concentration was adjusted to $1 \mu \mathrm{g}^{-1}$ before transfection. S2 cells were transfected with Flag-tagged 14-3-3 and V5-tagged Tctp (or V5tagged Rheb) using Effectene reagent (Qiagen). After $24 \mathrm{~h}$ of transfection, $0.1 \mathrm{M}$ $\mathrm{CuSO}_{4}$ was added to final concentration $0.5 \mathrm{mM}$ to induce protein expression using pMT vector. S2 cells were incubated for additional $48 \mathrm{~h}$, and extracted using CHAPS-based lysis buffer (0.1\% CHAPS, $20 \mathrm{mM}$ HEPES, $1 \mathrm{mM}$ EDTA, $1 \mathrm{mM}$ PMSF and a protease inhibitor cocktail (Roche)). Other steps were carried out according to the described protocol ${ }^{52}$. In brief, $50 \mu \mathrm{l}$ of $\mathrm{G}$-sepharose beads was washed and incubated with $2 \mu \mathrm{l}$ of anti-Flag antibody (Sigma 1,804) in $500 \mu \mathrm{l}$ of CHAPS-based lysis buffer for $2 \mathrm{~h}$ at room temperature. S2 cell lysates were incubated with $20 \mu \mathrm{l}$ of G-sepharose beads to avoid non-specific binding between resin and protein extracts. The S2 cell lysates were then incubated with anti-Flagbound G-sepharose beads overnight at $4^{\circ} \mathrm{C}$. After three times of 5-min washing with CHAPS-based lysis buffer, $20 \mu \mathrm{l}$ of lysis buffer was mixed with $5 \mu \mathrm{l}$ of $5 \times$ SDS loading buffer. The samples were boiled at $94^{\circ} \mathrm{C}$ for $5 \mathrm{~min}$ and centrifuged at 12,000 r.p.m for $5 \mathrm{~min}$. Twenty microlitre of each sample was loaded on SDS gel for western blotting. Mouse anti-V5 (1:5,000, Invitrogen 46-0705) was used to detect V5-Tctp or V5-Rheb. Co-IP between V5myc-Rheb and V5-Tctp was carried out using anti-Myc (Abcam Ab9106) as bait to immunoprecipitate V5myc-Rheb. Co-immunoprecipitated V5-Tctp was detected by anti-Tctp antibody. Immunoprecipitated V5myc-Rheb was detected with rabbit anti-Myc at $1: 5,000$.

RNAi treatment of S2 cells. S2 cells were treated with dsRNA following the protocol by Rogers et al. ${ }^{53}$ with minor modifications. Primers used for $14-3-3 \varepsilon$ RNAi were $5^{\prime}$-TAATACGACTCACTATAGGGAGAAATGGTGGAGGCCATG AAG $-3^{\prime}$ and $5^{\prime}$-TAATACGACTCACTATAGGGAGATTCAATGCCAAGCCCA AAC- $3^{\prime}$. Primers used for $14-3-3 \zeta$ RNAi were $5^{\prime}$-TAATACGACTCACTATAGGC GAAGCATCCGCTAGAAAACAGC- $3^{\prime}$ and $5^{\prime}$-TAATACGACTCACTATAGGTC GTTCAGTGTGTCCAGCTCGG-3'. Primers were designed using SnapDragondsRNA design (http://www.flyrnai.org/snapdragon). PCR reactions were carried out to amplify $\sim 500 \mathrm{bp}$ target sequence of $14-3-3$. Reverse transcription reaction was performed using MEGAscript T7 (Ambion) and the products were purified with MEGAClear (Ambion). S2 cells cultured in M3 media (Sigma) with $10 \%$ insect medium supplement (Sigma) were seeded on 12-well plate with the density of $1 \times 10^{6}$ cells per ml. For 14-3-3 RNAi treatment, RNA for $14-3-3 \varepsilon$ and $14-3-3 \zeta$ was added to media at 5 and $10 \mu \mathrm{g} \mathrm{ml}^{-1}$, respectively. After 3 days from the first treatment, RNAs were added the second time on fourth day. S2 cells were collected each day and lysed in $200 \mu \mathrm{l}$ of cold CHAPS-based lysis buffer for $30 \mathrm{~min}$ at $4{ }^{\circ} \mathrm{C}$ with rotating. Cell extracts were centrifuged at 12,000 r.p.m for $5 \mathrm{~min}$ at $4{ }^{\circ} \mathrm{C}$. Five micro-liter of $5 \times$ SDS loading buffer was added to $20 \mu$ of sample. Total amount of $25 \mu \mathrm{l}$ sample was boiled at $94^{\circ} \mathrm{C}$ for $5 \mathrm{~min}$, and $20 \mu \mathrm{l}$ sample was loaded for western blot analysis. The following antibodies were used at the indicated dilution: rabbit anti-14-3-3 (Invitrogen 51-0700) at 1:1,000, rabbit anti-Tctp ${ }^{8}$ at 1:2,500; rat anti-Rheb at 1:500, and mouse anti- $\beta$-actin (Abcam Ab8224) at 1:5,000. For detecting S6k and phospho-S6k, mouse anti-S6k (ref. 8) and rabbit anti-pS6k (Thr 398) (Cell signaling 9209S) were used at the same 1:1,000 dilution, respectively. For detecting Thor protein and phosphorylated Thor, rabbit anti-Thor antibody ${ }^{54}$ and rabbit anti-pThor (Thr 37/46) (Cell Signaling 2855S) were used at $1: 5,000$ and 1:1,000, respectively.

All uncropped western blots can be found in Supplementary Fig. 7.

\section{References}

1. Laplante, M. \& Sabatini, D. M. mTOR signaling at a glance. J. Cell Sci. 122, 3589-3594 (2009).

2. Inoki, K., Li, Y., Zhu, T. Q., Wu, J. \& Guan, K. L. TSC2 is phosphorylated and inhibited by Akt and suppresses mTOR signalling. Nat. Cell Biol. 4, 648-657 (2002).

3. Inoki, K., Li, Y., Xu, T. \& Guan, K. L. Rheb GTPase is a direct target of TSC2 GAP activity and regulates mTOR signaling. Genes Dev. 17, 1829-1834 (2003).

4. Garami, A. et al. Insulin activation of Rheb, a mediator of mTOR/S6K/4E-BP signaling, is inhibited by TSC1 and 2. Mol. Cell 11, 1457-1466 (2003).

5. Montagne, J. et al. Drosophila S6 kinase: a regulator of cell size. Science 285, 2126-2129 (1999).

6. Patel, P. H. et al. Drosophila Rheb GTPase is required for cell cycle progression and cell growth. J. Cell Sci. 116, 3601-3610 (2003).

7. Zhang, H., Stallock, J. P., Ng, J. C., Reinhard, C. \& Neufeld, T. P. Regulation of cellular growth by the Drosophila target of rapamycin dTOR. Genes Dev. 14, 2712-2724 (2000)

8. Hsu, Y. C., Chern, J. J., Cai, Y., Liu, M. \& Choi, K. W. Drosophila TCTP is essential for growth and proliferation through regulation of dRheb GTPase. Nature 445, 785-788 (2007).

9. Kim, D. K. et al. Translationally controlled tumour protein is associated with podocyte hypertrophy in a mouse model of type 1 diabetes. Diabetologia 55, 1205-1217 (2012).

10. Kobayashi, D. et al. Translationally controlled tumor protein is a novel biological target for neurofibromatosis type 1-associated tumors. J. Biol. Chem 289, 26314-26326 (2014).

11. Berkowitz, O., Jost, R., Pollmann, S. \& Masle, J. Characterization of TCTP, the translationally controlled tumor protein, from Arabidopsis thaliana. Plant Cell 20, 3430-3447 (2008).

12. Dong, X., Yang, B., Li, Y., Zhong, C. \& Ding, J. Molecular basis of the acceleration of the GDP-GTP exchange of human ras homolog enriched in brain by human translationally controlled tumor protein. J. Biol. Chem. 284, 23754-23764 (2009).

13. Brioudes, F., Thierry, A. M., Chambrier, P., Mollereau, B. \& Bendahmane, M. Translationally controlled tumor protein is a conserved mitotic growth integrator in animals and plants. Proc. Natl Acad. Sci. USA 107, 16384-16389 (2010).

14. Koziol, M. J. \& Gurdon, J. B. TCTP in development and cancer. Biochem. Res. Int. 2012, 105203 (2012)

15. Nagano-Ito, M. \& Ichikawa, S. Biological effects of Mammalian translationally controlled tumor protein (TCTP) on cell death, proliferation, and tumorigenesis. Biochem. Res. Int. 2012, 204960 (2012).

16. Hong, S. T. \& Choi, K. W. TCTP directly regulates ATM activity to control genome stability and organ development in Drosophila melanogaster. Nat. Commun. 4, 2986 (2013)

17. Zhang, J. et al. Role of the translationally controlled tumor protein in DNA damage sensing and repair. Proc. Natl Acad. Sci. USA 109, E926-E933 (2012).

18. Tuynder, M. et al. Biological models and genes of tumor reversion: cellular reprogramming through tpt1/TCTP and SIAH-1. Proc. Natl Acad. Sci. USA 99, 14976-14981 (2002).

19. Amson, R., Pece, S., Marine, J. C., Di Fiore, P. P. \& Telerman, A. TPT1/ TCTPregulated pathways in phenotypic reprogramming. Trends Cell Biol. 23, 37-46 (2013).

20. Fonseca, B. D., Smith, E. M., Lee, V. H., MacKintosh, C. \& Proud, C. G. PRAS40 is a target for mammalian target of rapamycin complex 1 and is required for signaling downstream of this complex. J. Biol. Chem. 282, 24514-24524 (2007).

21. Li, Y., Inoki, K., Yeung, R. \& Guan, K. L. Regulation of TSC2 by 14-3-3 binding. J. Biol. Chem. 277, 44593-44596 (2002).

22. Cai, S. L. et al. Activity of TSC2 is inhibited by AKT-mediated phosphorylation and membrane partitioning. J. Cell Biol. 173, 279-289 (2006).

23. Nascimento, E. B. et al. Phosphorylation of PRAS40 on Thr246 by PKB/AKT facilitates efficient phosphorylation of Ser183 by mTORC1. Cell Signal. 22, 961-967 (2010). 
24. Gwinn, D. M. et al. AMPK phosphorylation of raptor mediates a metabolic checkpoint. Mol. Cell 30, 214-226 (2008).

25. Fu, H., Subramanian, R. R. \& Masters, S. C. 14-3-3 proteins: structure, function, and regulation. Annu. Rev. Pharmacol. Toxicol. 40, 617-647 (2000).

26. Morrison, D. K. The 14-3-3 proteins: integrators of diverse signaling cues that impact cell fate and cancer development. Trends Cell Biol. 19, 16-23 (2009).

27. Pallares-Cartes, C., Cakan-Akdogan, G. \& Teleman, A. A. Tissue-specific coupling between insulin/IGF and TORC1 signaling via PRAS40 in Drosophila. Dev. Cell 22, 172-182 (2012).

28. Skoulakis, E. M. \& Davis, R. L. Olfactory learning deficits in mutants for leonardo, a Drosophila gene encoding a 14-3-3 protein. Neuron 17, 931-944 (1996).

29. Acevedo, S. F., Tsigkari, K. K., Grammenoudi, S. \& Skoulakis, E. M. In vivo functional specificity and homeostasis of Drosophila 14-3-3 proteins. Genetics 177, 239-253 (2007).

30. Chang, H. C. \& Rubin, G. M. 14-3-3 epsilon positively regulates Ras-mediated signaling in Drosophila. Genes Dev. 11, 1132-1139 (1997).

31. Kockel, L., Vorbruggen, G., Jackle, H., Mlodzik, M. \& Bohmann, D. Requirement for Drosophila 14-3-3 zeta in Raf-dependent photoreceptor development. Genes Dev. 11, 1140-1147 (1997).

32. Nielsen, M. D., Luo, X., Biteau, B., Syverson, K. \& Jasper, H. 14-3-3 Epsilon antagonizes FoxO to control growth, apoptosis and longevity in Drosophila. Aging cell 7, 688-699 (2008).

33. Dong, J. et al. Elucidation of a universal size-control mechanism in Drosophila and mammals. Cell 130, 1120-1133 (2007).

34. Oh, H. \& Irvine, K. D. In vivo regulation of Yorkie phosphorylation and localization. Development 135, 1081-1088 (2008).

35. Su, T. T. et al. Cell cycle roles for two 14-3-3 proteins during Drosophila development. J. Cell Sci. 114, 3445-3454 (2001).

36. Brand, A. H. \& Perrimon, N. Targeted gene expression as a means of altering cell fates and generating dominant phenotypes. Development 118, 401-415 (1993).

37. Inoki, K., Li, Y., Xu, T. \& Guan, K. L. Rheb GTPase is a direct target of TSC2 GAP activity and regulates mTOR signaling. Genes Dev. 17, 1829-1834 (2003).

38. Manning, B. D. \& Cantley, L. C. Rheb fills a GAP between TSC and TOR. Trends Biochem. Sci. 28, 573-576 (2003).

39. Wullschleger, S., Loewith, R. \& Hall, M. N. TOR signaling in growth and metabolism. Cell 124, 471-484 (2006).

40. Boer, K. et al. Gene expression analysis of tuberous sclerosis complex cortical tubers reveals increased expression of adhesion and inflammatory factors. Brain Pathol. 20, 704-719 (2010).

41. Lowe, S. W. \& Lin, A. W. Apoptosis in cancer. Carcinogenesis 21, 485-495 (2000).

42. Shah, O. J., Wang, Z. \& Hunter, T. Inappropriate activation of the TSC/Rheb/ mTOR/S6K cassette induces IRS1/2 depletion, insulin resistance, and cell survival deficiencies. Curr. Biol. 14, 1650-1656 (2004).

43. Cai, S. L. et al. Activity of TSC2 is inhibited by AKT-mediated phosphorylation and membrane partitioning. J. Cell Biol. 173, 279-289 (2006).

44. Hu, F. \& Liu, F. Targeting tissue-specific metabolic signaling pathways in aging: the promise and limitations. Protein Cell 5, 21-35 (2014).

45. Dong, J. \& Pan, D. Tsc2 is not a critical target of Akt during normal Drosophila development. Genes Dev. 18, 2479-2484 (2004).

46. Banerjee, S., Crouse, N. R., Emnett, R. J., Gianino, S. M. \& Gutmann, D. H. Neurofibromatosis-1 regulates mTOR-mediated astrocyte growth and glioma formation in a TSC/Rheb-independent manner. Proc. Natl Acad. Sci. USA 108, 15996-16001 (2011)
47. Yarm, F. R. Plk phosphorylation regulates the microtubule-stabilizing protein TCTP. Mol. Cell Biol. 22, 6209-6221 (2002).

48. Maeng, J., Kim, M., Lee, H. \& Lee, K. Insulin induces phosphorylation of serine residues of translationally controlled tumor protein in 293T cells. Int. J. Mol. Sci. 16, 7565-7576 (2015).

49. Zheng, M. et al. Inactivation of Rheb by PRAK-mediated phosphorylation is essential for energy-depletion-induced suppression of mTORC1. Nat. Cell Biol. 13, 263-272 (2011).

50. Yaffe, M. B. How do 14-3-3 proteins work?-- Gatekeeper phosphorylation and the molecular anvil hypothesis. FEBS Lett. 513, 53-57 (2002).

51. Xu, T. \& Rubin, G. M. Analysis of genetic mosaics in developing and adult Drosophila tissues. Development 117, 1223-1237 (1993).

52. Ceriani, M. F. Coimmunoprecipitation on Drosophila cells in culture. Methods Mol. Biol. 362, 423-427 (2007).

53. Rogers, S. L. \& Rogers, G. C. Culture of Drosophila S2 cells and their use for RNAi-mediated loss-of-function studies and immunofluorescence microscopy. Nat. Protoc. 3, 606-611 (2008).

54. Miron, M. et al. The translational inhibitor $4 \mathrm{E}-\mathrm{BP}$ is an effector of $\mathrm{PI}(3) \mathrm{K} / \mathrm{Ak}$ signalling and cell growth in Drosophila. Nat. Cell Biol. 3, 596-601 (2001).

\section{Acknowledgements}

We are grateful to Drs Kyung-Ok Cho and Efthimios Skoulakis for critical comments on the manuscript, and to Dr Nahum Sonenberg for generous sharing of anti-Thor antibody. We acknowledge the Bloomington Drosophila Stock Center, the National Institute of Genetics stock center, the Vienna Drosophila Resource Center, the Drosophila Genomics Resource Center and the Developmental Studies Hybridoma Bank for fly stocks and antibodies. This research was supported by a National Research Laboratory grant (NRF-2011-0028326) and a Global Research Laboratory grant (2014K1A1A2042982) through the National Research Foundation of Korea, funded by the Korean, Ministry of Education, Science \& Technology.

\section{Author contributions}

Y-C.H. and K-W.C made the initial observation of genetic interaction; T.P.L. and K-W.C designed the experiments. L.T.V. performed co-IP assays. A-R.K. and T.P.L. generated anti-Rheb antibody. T.P.L. performed all the other experiments; T.P.L. and K-W.C analysed the data and wrote the paper.

\section{Additional information}

Supplementary Information accompanies this paper at www.nature.com/nature.

Competing financial interests: The authors declare no competing financial interests.

Reprints and permission information is available online at http://npg.nature.com/ reprintsandpermissions/

How to cite this article: Le, T. P. et al. 14-3-3 proteins regulate Tctp-Rheb interaction for organ growth in Drosophila. Nat. Commun. 7:11501 doi: 10.1038/ncomms11501 (2016)

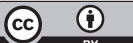

This work is licensed under a Creative Commons Attribution 4.0 International License. The images or other third party material in this article are included in the article's Creative Commons license, unless indicated otherwise in the credit line; if the material is not included under the Creative Commons license, users will need to obtain permission from the license holder to reproduce the material To view a copy of this license, visit http://creativecommons.org/licenses/by/4.0/ 